\title{
Captures
}

Figures, théories et pratiques de l'imaginaire

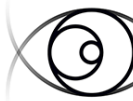

C A P

\section{Lire en mode conflictuel}

Non-fiabilité et indécidabilité, l'exemple du Black Note de Tanguy Viel

\section{Cassie Bérard}

Volume 3, Number 2, November 2018

\section{Littérature suspecte}

URI: https://id.erudit.org/iderudit/1055826ar

DOI: https://doi.org/10.7202/1055826ar

See table of contents

Publisher(s)

Figura, Centre de recherche sur le texte et l'imaginaire

ISSN

2371-1930 (digital)

Explore this journal

Cite this article

Bérard, C. (2018). Lire en mode conflictuel : non-fiabilité et indécidabilité, l'exemple du Black Note de Tanguy Viel. Captures, 3(2).

https://doi.org/10.7202/1055826ar

\section{Article abstract}

Starting from the principle that unreliable narrations in novels encourage the reader to produce decisive readings, this article attempts to grasp the process by which fictional universes are reproduced, thus creating the image of the narrators. It also aims to explore a particular mode of narrative reading: that of conflict. Where the reader accepts the unreliability in narrative in order to make it the place of an undecided reading, all possibilities are reconciled.
This document is protected by copyright law. Use of the services of Erudit (including reproduction) is subject to its terms and conditions, which can be viewed online.

https://apropos.erudit.org/en/users/policy-on-use/ 


\section{Lire en mode conflictuel}

\section{Non-fiabilité et indécidabilité, l'exemple du Black Note de Tanguy Viel Cassie Bérard}

Résumé :

Partant de l'idée que les narrations non fiables dans les romans encouragent le lecteur à produire des lectures décisives, l'article cherche à saisir le processus par lequel s'effectue la reconstruction des univers fictionnels et se forge l'image des narrateurs. II vise par ailleurs à explorer un mode particulier de lecture narrative : celui du conflit. Là où le lecteur accepte la non-fiabilité au cœur du récit afin d'en faire le lieu d'une lecture indécise, il réconcilie tous les possibles.

Starting from the principle that unreliable narrations in novels encourage the reader to produce decisive readings, this article attempts to grasp the process by which fictional universes are reproduced, thus creating the image of the narrators. It also aims to explore a particular mode of narrative reading: that of conflict. Where the reader accepts the unreliability in narrative in order to make it the place of an undecided reading, all possibilities are reconciled.

On soupçonne un narrateur de faire preuve de non-fiabilité lorsque s'additionnent dans son discours des inconsistances, des maladresses, des contradictions, ou encore, lorsque la version des événements donnée par un autre personnage de la fiction le contredit. En ce sens, peu de lectures peuvent octroyer au docteur Sheppard d'autre rôle que celui de narrateur manipulateur quand il rend compte, par écrit, des circonstances du meurtre de son patient Roger Ackroyd dans le roman d'Agatha Christie (1992 [1926]), puisqu'au terme de l'enquête, Hercule Poirot, détective perspicace comme nul autre, lève le voile sur la tromperie : il accuse Sheppard et l'oblige (à l'aide d'une technique de chantage efficace) à avouer sa culpabilité. Un tel dénouement met au jour l'attitude retorse et les motivations frauduleuses du narrateur : dès le début de son récit, il s'appliquait à camoufler la vérité. Cette révélation venant d'un autre personnage, dont l'autorité policière, mais aussi de héros, influence évidemment la lecture, ne laisse plus d'ambiguïté à la fin du roman : le narrateur a trafiqué sa version des faits pour dissimuler son crime. Le lecteur est soulagé de bénéficier de l'ingéniosité de Poirot, qui lui évite d'être la dupe de la mauvaise foi du narrateur.

À première vue, cet exemple des plus célèbres ne souffre d'aucune équivoque sur le plan de l'enquête. Le résumé du récit, tel que je viens de le formuler, se plie à la lecture traditionnelle que le texte s'efforce de nous faire adopter. Or, c'est justement cette lecture fermée, voire toute lecture fermée, que veut décourager Pierre Bayard dans Qui a tué Roger Ackroyd? (1998), où il démontre l'impossible culpabilité de Sheppard et pointe le 
délire d'interprétation qui guide Poirot dans ses accusations. Bayard indique, ce faisant, que la lecture littéraire gagne à dépasser les évidences pour dévoiler ce qui, dans les textes, reste dissimulé (soit, ici, le véritable coupable). II montre, du coup, qu'une résolution n'est jamais qu'une résolution parmi d'autres.

C'est ce qui, selon Gregory Currie, caractérise les œuvres non fiables, à savoir la propriété qu'elles ont de placer le lecteur au carrefour de plusieurs interprétations. Les œuvres que Currie qualifie de non fiables opéreraient ainsi leur supercherie à partir des présupposés de la lecture (2004: 140-141). Cette supercherie prendrait deux formes (Currie: 140-141). Ou bien l'œuvre — sa partition la plus exposée — convaincrait le lecteur d'une version particulière des événements de la fiction, quand une lecture scrupuleuse de ses mécanismes permettrait d'en arriver à une plus probante version. Ou alors l'œuvre exposerait différentes versions des événements tout en laissant présupposer qu'une version a préséance sur les autres, sans offrir toutefois de résolution péremptoire. Ici, l'effet serait de pousser le lecteur à se former sa propre clôture du récit; une clôture bringuebalante, forcément. La nuance est mince qui distingue les deux formes d'œuvres non fiables relevées par Currie. En effet, ces formes ont en commun d'encourager le lecteur à statuer sur les événements de la fiction, favorisant leur fermeture, quand en fait le texte travaille leur ouverture.

Cette ouverture est un critère précieux pour penser de manière large le phénomène de non-fiabilité dans la fiction littéraire, d'autant plus lorsqu'il s'agit de s'intéresser strictement aux narrations. Ce que pointe Wayne C. Booth, lorsqu'il élabore une définition de la narration non fiable dans Rhetoric of Fiction (1961: 155-159), c'est, d'abord, l'écart de valeur ou de savoir entre le narrateur et l'auteur implicite - cette instance textuelle on ne peut plus clairvoyante qui en sait plus et le sait mieux que le narrateur -, mais aussi, par conséquent, le rejet, par le lecteur, d'une compréhension littérale du sens du texte au profit d'une interprétation qui vise à réparer ce qui ne se laisse pas saisir clairement dans cet écart. C'est dire que la lecture au premier degré des récits tenus par des narrateurs non fiables constitue un tremplin vers un sens second, plus subtil, auquel se raccroche le lecteur qui relève des incongruités dans les énoncés, entre les lignes, ou à propos d'autres de ses connaissances acquises, malmenées dans le texte. À ce degré « supérieur » de la lecture, on invoque, chez Vincent Jouve, la notion de « lecture narrative » (2006); chez Richard Saint-Gelais, celle de « lecture policière » (1997).

Il y a là, il me semble, une dynamique importante des narrations non fiables que l'on a tendance à oblitérer, considérant que nombre de théoriciens se sont trouvé, pour circonscrire la notion de non-fiabilité, à la compartimenter à outrance, fixant les narrateurs dans des cases et entraînant des lectures catégoriques. Ainsi, le lecteur s'adonne, avec le narrateur faillible, indigne de confiance, discordant, fou, idiot, clown, menteur, et ainsi de suite-1, à typer ce qui pourtant se prête au paradoxe, à l'ouverture. L'ouverture est telle, pour certaines narrations non fiables, qu'elle laisse place à des hypothèses de lecture irréconciliables. Aussi trouve-t-on dans 
Le meurtre de Roger Ackroyd, au terme d'analyses méticuleuses, les preuves de la culpabilité de Sheppard comme celles de son innocence; or il ne peut, en toute logique, qu'être coupable ou innocent. Une indécidabilité semblable est présente dans Le Black Note de Tanguy Viel, dont je soumets l'exemple dans cet article. C'est cette ouverture, cet espace de bifurcation — la lecture indécidable — que je me prépare effectivement à observer. Mon but est de rappeler que l'un des principaux attraits de la non-fiabilité dans les textes, et ce qui en fait un phénomène narratif si racoleur, est le soupçon avec lequel elle transige et qu'elle nourrit radicalement. De fait, par l'ambiguïté qui en constitue le premier fondement, la narration non fiable ne laisse pas de contrevenir à une logique nette du récit. Nulle parole ne peut être reçue sans méfiance dès lors que la crédibilité du narrateur est entachée; une hypothèse et son contraire peuvent advenir. La non-fiabilité, nous le verrons, institue un mode particulier de lecture narrative, celui du conflit. La mystification de la narration brouille parfois jusqu'aux plus fins détails des récits, elle rend la réalité fictionnelle impossible à retracer, contraignant le lecteur, à la recherche de la vérité de la fiction, à s'engager dans une entreprise policière, c'està-dire une lecture à indices « faite de rapprochements, de confrontations, voire de va-et-vient méfiants à travers le texte " (Saint-Gelais, 1997: 792), ou même à se plier, comme Pierre Bayard en accuse Hercule Poirot, au délire d'interprétation. Et donc, la lecture s'attardant à la narration, la narration étant portée par des contradictions, la lecture produit des versions de la fiction qui s'entrechoquent, voire s'excluent. C'est là que s'opère le conflit.

\title{
Reconstruction : lecture décidée, lecture indécise
}

\author{
II a toujours cru qu'on y croyait, parce qu'on acquiesçait, \\ on ne disait rien, et c'est nous au total qui le rendions \\ dupe, parce qu'on lui faisait croire qu'il disait vrai. \\ Tanguy Viel $\stackrel{2}{\text {. }}$
}

L'acte de transmission narrative est mis à mal dans les romans de la non-fiabilité. Des discours relevant notamment du faux témoignage, de l'exagération ou de la supercherie sont marqués par des stratégies qui fragilisent la crédibilité de la narration. Devant l'irrégularité ou l'inexactitude, c'est-à-dire les défaillances d'une parole, le lecteur exerce une critique sur le texte qui le place en mode reconstruction : sa lecture à indices vise à réparer la fiction. II s'active, coûte que coûte, et parfois au risque de passer à côté de l'expérience unique qu'il fait de ne pas être en mesure de trouver du sens à toute chose. Vincent Jouve théorise ce type de lecture narrative dans lequel le lecteur compense l'éclatement ou la polyphonie d'un texte par son travail d'inférence et de réflexion; ce lecteur, « [a]yant du mal à reconstruire une cohérence du contenu et ne parvenant plus à identifier la voix qui lui parle, [...] en vient à réfléchir sur ce qu'il vit personnellement dans sa relation au texte » (2006: 159). En l'absence de prise concrète sur la fiction, mis à distance de la voix qui raconte, le 
lecteur s'engage dans une avenue interprétative lui permettant de négocier avec le récit, d'en colmater les brèches. Ce faisant, il tâche de retrouver une certaine cohérence qui - si elle n'est plus dans les propos tenus — justifie à tout le moins le dysfonctionnement narratif. Or, ce besoin de justification ne se comble parfois qu'au moyen de contorsions, à la lecture, pour dénouer les fils entremêlés de l'histoire.

Aux manques trouant le récit se superposent les diagnostics du lecteur qui viennent apaiser un temps l'incertitude occasionnée, comme on le verra avec Le Black Note (1998), premier roman de l'écrivain Tanguy Viel. Meir Sternberg pointe des questions auxquelles fait face un lecteur qui actualise, au cours du processus de lecture, le champ de réalité représenté dans un texte, construisant et reconstruisant le monde fictionnel à partir des actions claires ou confuses de la trame narrative :

What is happening or has happened, and why? What is the connection between this event and the previous ones? What is the motivation of this or that character? To what extent does the logic of cause and effect correspond to that of everyday life? [...] Most of the answers to these questions, however, are not provided explicitly, fully and authoritatively [...] by the text, but must be worked out by the reader himself on the basis of the implicit guidance it affords. (Sternberg, 1978: 50.)

Le processus de reconstruction s'applique fort justement aux narrations qui donnent lieu à des récits en circonvolutions, tenus, la plupart du temps, par des narrateurs dont il convient à tout moment de s'interroger sur les motivations. Sternberg, en ce sens, distingue les trous dans les textes en fonction de la réponse qu'ils suscitent chez le lecteur. Certains trous, temporaires, se voient comblés partiellement ou entièrement au fil de la lecture, grâce aux signes et réponses que le texte met à la disposition du lecteur. Ce sont sur ces trous que misent la plupart des romans policiers, on l'aura compris. Les narrations non fiables, elles, creusent plutôt des trous permanents. Ceux-ci appellent chez le lecteur un investissement créatif des plus exigeants. De fait, le lecteur ne pourra, d'une part, s'assurer de la validité de ses diagnostics — lorsqu'il tente une hypothèse, il est fragilisé par son caractère invérifiable. II ne pourra, d'autre part et malgré lui, qu'effectuer une saisie partielle des « dits », mais surtout des « non-dits », de la narration. Ainsi s'établit tout diagnostic : sur la base d'une partition incomplète. Dans ce contexte, il m'apparaît que toute hypothèse de lecture « décidée " s'érige, en quelque sorte, pour contrer une indécidabilité dérangeante qui se trouve pourtant à être l'une des conditions intrinsèques de la non-fiabilité dans les textes.

Une indécidabilité caractérise ainsi la narration duBlack Note de Tanguy Viel, lequel s'avérera être un inconditionnel de l'ambiguïté narrative et des intrigues à magouilles (Cinéma, 1999; L'Absolue perfection du crime, 2001; Insoupçonnable, 2006; etc.). Le narrateur rend compte, dans un récit entrecoupé, dispersé et confus, des circonstances entourant la mort de son ami Paul. II tente de tracer le portrait de cet événement dramatique, mais surtout de reparcourir le chemin sinueux qu'ont emprunté pendant des 
années Georges, Elvin, Paul et lui-même pour devenir un quartet célèbre, sans succès. Ces quatre musiciens se sont installés sept ans auparavant sur une île, dans une maison qu'ils ont nommée le Black Note en hommage au label de jazz américain Blue Note. Habiter à quatre pour jouer de la musique, mais bientôt pour se perdre dans la drogue et la désillusion :

Dans cette maison, [...] c'est à force de vivre ensemble, non pas les uns sur les autres, non pas se frôlant entre deux portes dans cette maison, mais les uns contre les autres, se cognant dans les espaces vides, comme on se croisait trop souvent avec Paul, se cognant mentalement dans les yeux, à force on ne se supportait plus. (Viel, 1998: 16-17.)

Cette maison devenue mythique, lieu des rêves échoués, n'existe plus que par ses ruines et ses cendres à l'heure où le narrateur, interné dans un centre de désintoxication ou une clinique psychiatrique, ressasse cette époque où prévalait la vie commune. Le quartet lui-même s'est dissous : Elvin, confiné dans le même bâtiment que le narrateur, le croise dans le solarium sur le toit, où il profite de la vue. Georges, plus lucide aux dires du narrateur, s'est terré dans une « chambre de moine ». Et Paul est mort dans l'incendie criminel qui a anéanti le Black Note. Il est mort retenu à l'intérieur de la maison rongée par les flammes, mais, suppose le narrateur, « il s'éteignait [déjà] à force de l'héroïne dans le sang » (Viel, 1998: 16). Depuis, Paul hante le narrateur; il exerce sur lui une emprise étouffante. Dans cet esprit de ressassement, le récit du Black Note se construit autour d'un trou permanent, le crime, pourtant mis en évidence tout au long du roman. Restent à jamais incertaines, de fait, l'origine de l'incendie, ainsi que l'identité — voire l'intention — du responsable.

C'est à une dynamique de lecture conflictuelle que nous convie Tanguy Viel. La naïveté apparente du narrateur, que les bouffissures du style exposent, calfeutre certaines formules proches de la ruse, dessinant le caractère indécidable de la narration non fiable : « Et il y a des choses, Rudolph, plein de choses, on a toujours envie d'y croire, les pires aberrations on a envie d'y croire » (1998: 27). De fait, les marques discursives de la non-fiabilité sont multiples dans ce roman. Impossible de ne pas remarquer les répétitions décalées, les contradictions, les incohérences et invraisemblances dans le récit, puis la versatilité des allocutaires (Georges, Elvin, Paul et Rudolph, un pensionnaire). La scène d'énonciation reste indéterminée (on ne peut fixer clairement à qui s'adresse le narrateur $\underline{3}$ ). Le meurtre de Paul demeure irrésolu (la faute est jetée tour à tour par le narrateur sur les trois membres restants du quartet). Or, ce n'est pas sur la non-fiabilité que se porte la question de l'ambiguïté - il est évident que rien n'est clair —, mais sur la nature de cette non-fiabilité ou, pour le dire autrement, sur les motivations du narrateur, qui ne semble, paradoxalement, ni contrôler son discours ni en être complètement désinvesti. Le lecteur se trouve au carrefour d'au moins deux diagnostics de lecture qui ont chacun la potentialité d'influencer sa sympathie à l'égard du narrateur : fait-il preuve de bonne ou de mauvaise foi? Est-il inconsciemment non fiable ou trafique-t-il volontairement les faits? Le lecteur est alors 
tenté, supposé-je, d'offrir à ce dilemme une réponse décisive lui permettant de fermer à sa manière le récit, de lui offrir une cohérence par la résolution.

Le même débat moral présent chez Tanguy Viel entre inconscience et volonté se rencontre dansL'emploi du temps de Michel Butor (1956), où le narrateur, écrivant un journal personnel et se croyant rapidement victime (inconsciente) d'un complot orchestré par la ville dans laquelle il séjourne, laisse transparaître une rhétorique fourbe (volontaire) lorsqu'il dévoile, contre toute attente, la présence d'un destinataire à ses propos intimes. C'est manifestement ce même dilemme, traité différemment, que relève Meir Sternberg dans La Foire aux vanités (Vanity Fair) de William Thackeray tandis qu'elle oscille entre deux diagnostics de lecture (1978: 51) : Becky Sharp est-elle entreprenante (volontaire) ou manipulée et innocente (inconsciente) dans ses relations extraconjugales avec Lord Steyne? De semblables conflits, insolubles selon Sternberg, pointent les trous permanents dans les textes : « No reader can afford to disregard them, but he will look in vain for pat explicit answers. » (Sternberg: 51.)

Richard Saint-Gelais soutient, à cet égard, que le texte « n'est pas une instance absolument contraignante; il ne génère pas une lecture mais en permet plusieurs, parfois divergentes. Mais le texte n'est pas davantage une instance passive dont les lecteurs se disputeraient la vérité. " (1994: 75.) Le texte comporte une structure qui sous-tend des consignes de lecture et, de son côté, le lecteur en retient des éléments variés et y appose sa propre expérience, engendrant l'une ou l'autre des lectures « rentables ». En d'autres mots, “[l]e texte est une instance qui est à la fois travaillée par ses lectures et travaille celles-ci en retour en mettant en place des conditions d'exercice qui détermineront en partie leurs rentabilités respectives. 》 (Saint-Gelais, 1994: 75.) Les procédés textuels, en ce sens, participent à l'économie des lectures en configurant ce que Saint-Gelais nomme « des espaces rescripturaux de la lecture » (1994: 75). Voyant le texte comme un puzzle dont certaines pièces manquent, Saint-Gelais explicite une conception de la lecture qu'on retrouve également chez Wolfgang Iser : "The process of assembling the meaning of the text is not a private one, for although it does mobilize the subjective disposition of the reader, it does not lead to daydreaming but to the fulfillment of conditions that have already been structured in the text. » (1978: 49-50.) Ainsi, chaque lecteur, devant un puzzle incomplet, est amené à remplir — à sa manière tout en respectant les contours — les trous qui creusent le texte. À la lumière du mode de lecture conflictuel, je me permets cependant de reconfigurer légèrement la métaphore du cassetête. Je ne m'attarde pas aux pièces manquantes, mais m'imagine plutôt un casse-tête dysfonctionnel pour lequel seraient fournis plusieurs morceaux aux contours identiques. Tout encouragerait le lecteur à choisir parmi une banque de morceaux celui qui lui plaît (l'obligeant à délaisser les autres) pour remplir les espaces et compléter le puzzle. II reste toutefois intéressant de penser la persistance du conflit des lectures en rentabilisant cette métaphore : et si devant la multitude des images fournies pour combler les trous, le lecteur préférait opter pour le vide? Ou plutôt, pour une lecture qui accepterait de ne rien résoudre en admettant que la 
résolution se trouve dans le foisonnement des possibilités.

Les décisions, conscientes ou non, que prend le lecteur en fonction des indices dans le texte participent à une recréation de la fiction autrement réfléchie de lecture en relecture. Tenter d'associer tel événement décrit par le narrateur à telle pensée qu'il élabore plus tard, tenter d'établir des liens de causalité entre ses actions pêlemêle, de lire entre les lignes de son discours confus, dans le métatexte, voire le paratexte, histoire d'éclairer les zones troubles, cela comporte des risques interprétatifs. Ainsi, conclure à la démence du narrateur du Black Note et associer sa non-fiabilité inconsciente à l'effet néfaste des drogues sur son jugement ou, en revanche, pointer les motifs qui confirment son projet de quitter le centre et prendre ses détours rhétoriques pour des marques de manipulation aide bien sûr le lecteur à se constituer une instance narrative plus nette pour suppléer à son caractère fuyant. Or, ce processus diagnostic tend aussi à catégoriser le personnage, et par là, à en atténuer la complexité. Cela peut signifier - et je m'avance à dessein sur une pente glissante - que, dans sa configuration de la fiction, le lecteur est parfois plus satisfait devant la clarté du mirage que devant la dure expérience des nuances, ou l'angoisse du chaos.

\section{Première impression : l'arbitraire du commencement}

Comprendre qu'on ne peut pas vivre à l'infini sur des ruines, à l'infini sur des champs de bataille, tu comprends, ceux-là vidés d'âmes, les champs de bataille, et qu'on ne peut pas se faire une place entre deux lambeaux de chair. Tanguy Viel ${ }^{4}$.

Il est naturel de penser qu'en tant qu'expérience unique, chaque lecture repose sur des associations qui relient le monde fictionnel au monde du lecteur. De telles liaisons s'effectuent dès l'entrée dans le texte, voire dès le paratexte, et coordonnent d'emblée des configurations :

The reader of a text does not wait until the end before beginning to understand it, before embarking upon its semantic integration. [...] The reader tries to organize the so-far incomplete semantic material given him in the best possible way. [...] Of course, inferences in the initial stages of reading are necessarily tentative. (Perry, 1979: 46.)

Des fictions s'ouvrent, par leurs signes, à une pluralité de représentations possibles mises en marche par le lecteur et réactualisées tout au long de sa traversée du texte. Les configurations de la lecture se voient donc, parfois, contredites par des éléments qui, dans le texte, les ébranlent, ou par certaines inférences se développant, se renforçant, aux dépens d'autres. Dans bien des cas, remarque Menakhem Perry (47), le lecteur élabore, dès le début du texte, les hypothèses les plus rentables compte tenu des données 
insuffisantes dont il dispose; des hypothèses qu'il n'aurait certainement pas construites, du moins auxquelles il n'aurait jamais donné tant d'importance, s'il avait été en possession d'informations qui ne viendront que plus tard dans le processus de lecture. Ainsi, au fur et à mesure que progressent le récit et l'histoire, le lecteur raffine ses inférences en vue d'une compréhension optimale du texte et de ses composantes. De la même manière, les personnages, y compris le narrateur, sont l'objet, dès leur apparition, du jugement du lecteur :

we may build up at the beginning of the text a sympathetic or a balanced attitude towards a character, [...] with the text initially presenting only (or mainly) positive aspects of it. Even if some dubious qualities or actions of this character occur at the beginning of such a text, they are not prominent enough to be considered counter-instances - at most they present the character as a creditable person with a few weaknesses. (Perry, 1979: 47.)

Perry suppose que, la plupart du temps, le lecteur donne aux personnages de fiction le bénéfice du doute d'entrée de jeu. Ce n'est que plus tard, selon l'évolution des actions, que le personnage peut entraîner la méfiance du lecteur au point d'en perdre, dans certains cas, sa crédibilité. Nos conventions de lecture et nos appréhensions ont bel et bien subi quelques remous depuis la parution, en 1926, du roman d'Agatha Christie qui rappelait au large public - estomaqué par la supercherie - que même l'instance narrative n'est pas toujours digne de confiance. Depuis, le surréalisme, le Nouveau roman ont marqué les pratiques de lecture, l'ère du soupçon et l'ère déconstructiviste ont multiplié les expériences du doute, et combien de théories ont mis au jour les stratagèmes romanesques et accusé la mauvaise foi des auteurs? Le lecteur d'aujourd'hui ne peut plus être totalement la dupe de telles stratégies de mystification. Toutefois, peu différent sur d'autres points des lecteurs de tout temps, supposé-je, le lecteur d'aujourd'hui a naturellement besoin de stabilité, ce qui expliquerait son souci de donner du sens aux récits qu'il lit, qu'importe par quelles manœuvres d'accommodement il doit passer pour y arriver.

Je me permets d'imaginer, alors, un tel besoin chez le lecteur duBlack Note d'ajuster sa lecture en cours de route. Au commencement, le lecteur accorde une crédibilité légitime au narrateur se livrant avec une apparence de sincérité et d'humanité qui facilite l'adhésion : «C'est la façon qu'on a tous de se croiser dans le parc, dans les couloirs, il y a des liens très vite qui se créent. Ici, les gens, tu les aimes malgré toi. » (Viel, 1998: 11.) La surenchère et le désordre de la narration ne deviennent inquiétants, ou plutôt dérangeants, qu'un peu plus tard dans le premier chapitre, peut-être au moment où le narrateur, cessant momentanément de laisser transparaître sa vulnérabilité, se défend : «Moi aussi je vais mieux, ce n'est pas moi qui ai tout inversé, c'est Paul seulement, moi je n'ai jamais dépassé les limites. » (20.) Le lecteur est alors en mesure d'effectuer un pas de côté, de relativiser la parole du narrateur au vu de son changement d'attitude. II a un prétexte pour se tenir sur ses gardes; or, cela ne signifie pas qu'il le fait forcément. 
La «première impression » a une grande importance dans le parcours de lecture. Elle est des plus durables, explique Sternberg. Sa préséance, ajoute Perry (35), exerce un étonnant pouvoir de persuasion sur le lecteur. Celui-ci se construit rapidement une première image du narrateur homodiégétique qui s'engage, et par là s'expose, dans son récit. Cette image influencera, par la suite, la réception des différents éléments contenus dans le texte. La première impression, bien qu'elle puisse être ébranlée ou rejetée plus tard, continuera généralement d'avoir une incidence sur la compréhension du lecteur tout au long de sa lecture, constate Shlomith Rimmon-Kenan (1983: 121). Dans le même sens, Perry note cette tendance des individus à persister dans la direction dans laquelle ils se sont d'abord engagés, et ce, pour tout type d'activité (1979: 53). Une tendance tout autant observable dans l'activité de lecture. Perry associe ainsi « l'effet de primauté » (primacy effect) à la capacité de persuasion et à la ténacité de la première impression de lecture (53). Les informations transmises en premier dans un texte, suivant l'étude de Perry, sont décisives pour le portrait général qu'un lecteur se fait d'un personnage, et décisives, de même, pour les manœuvres d'accommodement qu'il entreprendra au fil de sa lecture.

Perry et Sternberg, dans leurs travaux respectifs (Perry: 55; Sternberg: 93), évoquent l'expérimentation scientifique d'Abraham S. Luchins (1957), chercheur en psychologie, pour illustrer l'attitude d'une audience qui a en main deux arguments de lecture opposés, soit « a conflicting information in one continuum » (Perry: 55). Deux groupes reçoivent dans un ordre inverse deux fiches d'informations sous forme de récit concernant un personnage nommé Jim. La fiche A décrit un personnage extraverti et amical; la fiche $B$, un personnage renfermé, peu amical. Bien que les caractéristiques soient contradictoires, elles sont transmises de manière à donner de l'information sur un même personnage. Les membres des deux groupes doivent témoigner, au terme de leurs lectures, de leur impression concernant Jim. Les résultats de l'expérience de Luchins démontrent une propension à reconnaître en Jim les traits de personnalité présentés en première instance. Quant aux informations reçues en second, elles seraient rejetées ou associées à des comportements circonstanciels, exceptionnels du personnage, que les lecteurs ne voient pas la nécessité de considérer comme importants (1979: 55). Par cette manœuvre interprétative se constitue, en quelque sorte, une mise à plat du personnage.

L'effet de primauté n'est toutefois pas déterminant dans toute expérience de lecture. Par exemple, le degré d'autorité ou la réputation de l'instance qui transmet l'information, explique Perry (54), peut influencer radicalement le diagnostic de lecture, qu'importe l'ordre de présentation. Hercule Poirot se prononce à la toute fin du Meurtre de Roger Ackroyd contre le docteur Sheppard, qu'il accuse d'assassinat et, par voie de conséquence, de mensonge narratif. Même si rien, jusque-là dans le roman, ne nous permet de douter du récit de Sheppard, sa version ne fait plus le poids devant la parole de l'enquêteur. Et pourtant, les preuves qu'il amène ne concurrencent pas sérieusement la version de Sheppard; Pierre Bayard, à ce propos, taxe 
l'enquêteur de délire paranoïaque dans Qui a tué Roger Ackroyd?, souvenons-nous. En fait, ce sont vraisemblablement les enquêtes que Poirot a menées avec succès dans ses aventures précédentes qui lui assurent une autorité presque inébranlable. Ainsi s'efface magiquement, avec le verdict final de Poirot, l'image de l'homme intègre que le lecteur s'est construite de Sheppard depuis le début de sa lecture.

Si Hercule Poirot n'était pas Hercule Poirot, tout à coup le récit de Sheppard et l'image qu'il nous révèle de luimême pourraient avoir préséance sur la parole de Poirot; il est à parier que la confiance du lecteur serait alors plus difficile à ébranler. D'autant que, même à la fin du roman, lorsque Sheppard livre la vérité tel que Poirot le lui ordonne, si nous demeurons attentifs aux réactions du docteur, il paraît innocent. Sheppard semble davantage céder à la menace d'un Poirot manipulateur qu'effectuer des aveux sérieux lorsqu'il exhibe, avec une froideur inhabituelle, sa responsabilité dans le meurtre. Pour qui s'est laissé prendre par un doute généralisé, cette révélation ne cadre pas : elle sent la fabrication. Si Poirot n'était pas Poirot, rien ne nous interdirait de croire qu'il est lui-même coupable et, par extension, qu'il a trafiqué, dans les dernières lignes du texte, le récit que nous lisons pour triompher dans son enquête.

Cette brève escapade chez Agatha Christie m'amène à interroger, on l'aura compris, l'arbitraire de la première impression, dans la mesure où celle-ci se constitue conséquemment à une configuration menée par l'artisan du récit. Puisque les récits, dans les cas qui nous intéressent, relèvent de narrateurs non fiables, on ne peut sérieusement négliger l'ordre de présentation, susceptible de s'ajouter aux stratégies discursives de mystification les plus retorses. Il est vrai, cependant, que « l'effet de primauté » offre une piste éclairante pour observer un phénomène de double diagnostic comme celui que met en marche Le Black Note, où il n'est pas question d'autre autorité engageante, a priori, que celle de la narration. De fait, Perry va plus loin en ce qui concerne les propositions contradictoires soumises à l'expérience de la lecture :

when two sides of a controversy or an argument are presented, the side presented first has the advantage. [...] Contradiction is essential in such studies since their purpose is to get an unequivocal view as to which of the two parts of the message is more effective in determining the final impression. (1979: 53-54.)

Le conflit moral mettant en cause le narrateur du roman de Tanguy Viel propose en effet une controverse qui dépend beaucoup de l'ordre dans lequel surviennent ses confidences. Au terme de la lecture, si le lecteur doit évaluer l'implication du narrateur dans l'incendie criminel qui a coûté la vie à Paul, il lui demeure impossible de valider sa réponse avec certitude. Bien sûr, le narrateur avoue son crime à Rudolph, l'un de ses allocutaires, vers la fin du récit : «De savoir maintenant que j'ai mis le feu, je ne voulais pas te le dire, tu comprends, je ne voulais pas te mentir non plus, c'était seulement construire du neuf avec du vieux. » (Viel, 1998: 87.) Mais cet aveu, en fin de parcours, n'a pas plus de consistance que tous les aveux précédents où le narrateur jure de 
son innocence, ou que tous les aveux suivants, où il prétend avoir menti lorsqu'il confiait avoir mis le feu à la maison. Frank Wagner observe, à propos du Black Note, que ce sont les codes de la communication, la séparation ambiguë entre allocutaire et auditeur, qui entraînent, sur le plan de la lecture, des problèmes d'interprétation et génèrent des effets contradictoires :

D'une part en effet cette disparition des allocutaires peut tendre à accroître l'impact de la révélation finale sur le lecteur, car l'aveu du meurtre de «Paul » alias « John » par le narrateur-personnage est ainsi délivré dans une immédiateté éminemment crédibilisante : les relais intradiégétiques que représentaient les allocutaires successifs ont désormais disparu, de sorte que le lecteur peut avoir l'impression de bénéficier ainsi d'un accès direct à la « vérité » — enfin dévoilée sans détours. [...] Mais, d'autre part, cette mise à nu des codes énonciatifs peut être interprétée dans une perspective inverse, qui y identifierait une révélation métatextuelle de l'artifice du récit, à valeur de suggestion de fictionnalité. (2005: 238.)

Sur le plan de la non-fiabilité, les dysfonctionnements au sein de la narration dépassent la question de la culpabilité, car elles affectent la crédibilité de l'instance qui, elle seule, nous permet d'accéder à l'univers de fiction. L'échec de la transmission narrative est au premier plan; il devient ainsi le principal facteur d'influence de notre jugement à l'égard du personnage et de toute la fiction. Au même titre que Jim, dans l'étude de Luchins, est pour certains lecteurs amical et pour d'autres asocial - deux caractéristiques mutuellement exclusives -, le narrateur du Black Note est ou bien un être désillusionné, ou alors il est en plein contrôle de ses moyens : en train d'illusionner le lecteur, de lui jeter de la poudre aux yeux — et alors jusqu'où s'étend le mensonge? Son instabilité psychologique affecte la validité de sa narration sans qu'il en ait conscience (ce qui atteste sa vulnérabilité) ou alors les traces de faillibilité dans son discours sont un masque dont il se voile pour arriver à ses fins... L'inconscience ou la volonté derrière la non-fiabilité de la narration, répondant chacune à une partition trouée de la lecture, ne tracent pas une même image du narrateur, on l'aura deviné. Difficilement complémentaires, elles relèvent de motivations antithétiques. Dès lors, la responsabilité du narrateur n'a plus le même poids d'une version à l'autre. Dès lors, l'univers de fiction n'est plus reconstruit, à la lecture, selon les mêmes termes. Et pourtant, il s'agit bien, encore, d'un même récit.

L'expérimentation de Luchins déforme évidemment l'expérience de la lecture. On sait qu'un roman, sauf exception, se donne comme structure préalablement établie; les informations suivent un ordre qui est celui du récit. Aussi, il n'y a pas d'inversion des arguments d'une lecture à l'autre - pas de fiches $A$ et $B$ interchangeables —, de sorte que la controverse qui s'installe tout au long du texte fictionnel trouve bel et bien à s'actualiser au niveau de la lecture linéaire. II n'en demeure pas moins que nous pouvons imaginer un ordre différent que le narrateur du Black Note, dans d'autres circonstances, aurait pu préférer pour ménager les 
effets de son discours. Dans la partition actuelle du récit, le narrateur identifie Paul, très tôt, comme principal responsable de son propre malheur. Dans une partition virtuelle possible, le narrateur ouvrirait son récit en avouant son crime à Rudolph, comme il s'y prête, en fait, à la fin du roman. Étonnamment — nous arrivons à le concevoir par l'exemple - , le renvoi de la faute sur un homme mort qui ne peut témoigner ne dessine pas un narrateur aussi honnête que l'autoaccusation. Étonnamment encore, l'autoaccusation portée en fin de récit ne dessine pas un narrateur aussi honnête que si elle ouvrait le roman, que si les fautes étaient exposées d'emblée sans masque, que si l'entente avec le lecteur reposait sur ce partage de l'atrocité.

D'une certaine façon, tout le parcours de lecture se réclame de cet arbitraire qui est associé à la première impression. La compréhension de la narration du Black Note, ainsi, se fait en dent-de-scie; le lecteur hésitant entre une hypothèse et l'autre subit les humeurs fluctuantes d'une narration qui avance en se niant. Elle peut aussi souffrir un changement radical d'interprétation : on reconnaît d'abord les tourments tranquilles du narrateur pour suspecter plus tard sa sournoiserie. Mais la force persuasive de l'impression première peut expliquer quant à elle des comportements de lecture singuliers, tout autant de contorsions interprétatives au profit d'une lecture fermée : 1) que l'on s'explique des attitudes contradictoires du narrateur comme de simples écarts épisodiques; 2) que l'on oblitère complètement des données du texte qui contredisent une hypothèse; 3) que l'on repousse abusivement les limites du texte dans la recréation interprétative, et ce, jusqu'au délire paranoïaque. Et donc, au final, que l'on passe outre au conflit inhérent pour se soumettre à une lecture « décidée », alors même que le texte ainsi que son narrateur restent indécis ou imprécis jusqu'au bout.

\section{La vérité sur Le Black Note : du conflit à la réconciliation}

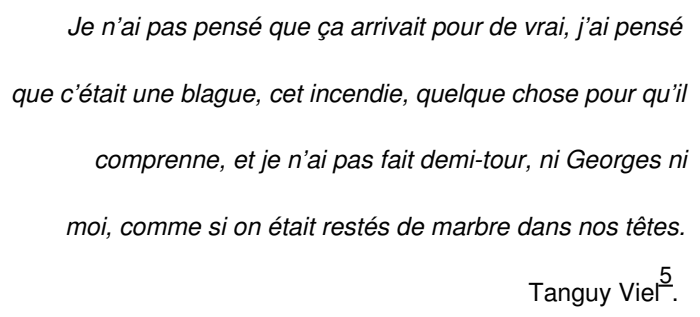

Dans Le Black Note, la confusion affecte la narration autant que l'intrigue; on peut parler d'une ambiguïté générale, où les différentes composantes de l'œuvre se phagocytent. L'agonie du quartet et le drame de l'incendie sont contés de manière fragmentée par la parole urgente et le regard partial d'un narrateur instable. Idées en boucles et fracturées, phrases cherchant leur sujet; le récit refuse la linéarité et l'enchaînement des actions. Se fait jour une narration répétitive et des formulations alambiquées qui reproduisent le mouvement houleux d'une mer violente prête à avaler un corps, ou d'un esprit tourmenté perdant contact avec la réalité. Le narrateur du Black Note s'émeut d'un passé qui lui permettait de croire en ses rêves, puis se satisfait du calme d'un endroit où Paul, enfin, n'exerce plus sur lui le contrôle de jadis. 
Indécis, il erre entre le présent las et le passé morbide. II hésite entre plusieurs versions des événements. II module son discours au gré des allocutaires : avec Georges, le partage des souvenirs, la nostalgie; avec Elvin, le discours paternaliste; avec Rudolph, le ton de la confession. C'est un narrateur aussi multiple que fuyant. II incarne l'éclatement.

Un tel éclatement, qu'aucune règle ne dicte, traduit une énonciation complexe, difficile à circonscrire. La structure du récit - l'enchaînement désordonné des idées puis des allocutaires — n'est pas en mesure de représenter une situation énonciative réaliste; elle s'apparente, en cela, au monologue intérieur, mais plus encore, à une forme de psychose. Les conditions d'enfermement du narrateur, après tout, laissent craindre une certaine folie. À cela s'ajoutent les visions de Paul, à qui le narrateur s'adresse comme à Georges et à Elvin, en les prenant à partie dans sa tête. Pourtant, il manie et remanie ses discours, s'interdit d'aller au bout de sa pensée, contrecarre s'il le faut ses propres aveux compromettants. II ajuste son témoignage en fonction de ses destinataires, bien que ceux-ci, par leur silence, ne semblent exister qu'en fantasmes. Imprécise, la scène d'énonciation accueille l'indécidabilité de la lecture; elle permet à la non-fiabilité de se déployer en dehors de réelles compromissions.

On l'a vu, la narration duBlack Note, parce qu'elle ne se fixe pas, parce qu'elle ne fait pas advenir la vérité, parce qu'elle s'organise autour de trous permanents, génère au moins deux diagnostics de lecture de la nonfiabilité, et parce qu'elle oscille entre révélation et dissimulation, rend durable l'indécidabilité. L'impuissance du narrateur, victime ayant basculé dans le revers du réel à cause des drogues, à cause des drames, montre un homme faillible. La folie qui le gagne laisse croire à une plongée inconsciente dans l'obscur abîme des songes; s'il en vient à inventer son crime, c'est, pouvons-nous penser, parce qu'il est rongé par un sentiment de culpabilité et parce qu'il n'arrive plus à tracer la limite entre fiction et réalité : « Même quand on n'a rien fait, on croit toujours qu'on est coupable quand on est trop proche, même quand on n'a rien fait. » (Viel, 1998: 87.) Dans sa typologie où elle distingue les narrateurs faillibles des narrateurs indignes de confiance, Greta Olson suppose qu'il y a matière, avec les premiers, à développer une empathie, car ils se présentent au lecteur affaiblis par des forces extérieures ne dépendant pas d'eux. C'est ainsi que le narrateur pris de folie du Black Note se laisse vaincre par le désordre et l'irrationnel. Dénoncer un crime ou renoncer — ce qu'il fait à tort et à travers tout au long du récit —, un tel narrateur est-il réellement capable de comprendre les incidences de ses choix?

La folie explique tout, conforte le lecteur et apaise les doutes. Cependant, pour qui la conçoit comme un artifice, elle côtoie subtilement la feinte; elle s'apparente à une stratégie d'évitement. Dès lors, le récit appuyé sur cette feinte donne raison au lecteur de douter de la bonne foi du narrateur. Ce dernier démontre un besoin de se confier, d'expulser ses hantises et ses fautes. Or, quel objectif motive réellement ces confidences? Le 
texte ne précise pas l'information. L'intrigue du Black Note, par ailleurs, se construit autour d'une affaire criminelle ayant, forcément, a une incidence sur l'acte de réception. C'est la «morbidité du "prétexte" narratif : le meurtre » qui, écrit Frank Wagner, sollicite « une réception de type projectif et participatif [...], puisque le lecteur aiguillonné par le désir de voir et de savoir est ainsi invité à suspendre momentanément son incrédulité et à adhérer à l'univers du récit » (2005: 241-242). Toutefois, dans le travail participatif du lecteur, c'est aussi ce même prétexte du meurtre qui active la méfiance à l'égard de la narration. L'implication personnelle du narrateur-personnage dans les événements et sa sensibilité vis-à-vis de leurs conséquences ne peuvent, vraisemblablement, être négligées puisqu'elles cadrent le récit. II donne tant d'importance à la mort de Paul qu'il contribue lui-même à créer un suspense et à susciter l'inquiétude. Par les détails compromettants qu'il livre à propos de l'incendie, il attire paradoxalement l'attention sur les informations qu'il ne livre pas, sur le savoir retenu (Fortier et Mercier, 2001) ou, par ailleurs, le savoir remanié. Plus aucune version n'est valide à la fin du récit, le narrateur le signale explicitement : il se blâme de n'avoir su faire que cela, mentir, malgré lui, tout au long des dernières années. Comment, suivant de tels énoncés, ne pas penser la narration en termes d'artifice? Comment ne pas entrevoir la possibilité d'une vaste dissimulation? Le narrateur, dans cette perspective, laisserait filer la vérité pour aussitôt en appeler au mensonge afin de l'enterrer. Rudolph, le pensionnaire à qui le narrateur se confie, devait emporter ses aveux de culpabilité avec lui en quittant définitivement la clinique. Mais il est de retour contre toute attente, et soudain, le narrateur sent le risque que cela implique pour ses propres espoirs de libération. Ses confessions à Rudolph suivent ce mouvement de ressac : il livre ses secrets puis les récupère. II répare, il tresse, il atténue. Georges coupable, Elvin effrayé, et lui, pris au piège entre les deux. Pris dans le nœud du conflit.

La vérité à propos du Black Note, c'est qu'il existe au moins une autre façon de lire les choses. La vérité, c'est que le conflit n'en est pas vraiment un. II s'agit plutôt, on l'aura compris, d'une réconciliation, une manière de nuancer la lecture trop facilement catégorique. Comme pour l'expérience de Luchins, où on révèle le caractère excentrique de Jim et son côté introverti, où on le dessine comme un être aimable et, alternativement, asocial, les narrateurs non fiables ne sont pas des figures univoques. Penser ces narrateurs en termes de types, tel que nous enjoint à le faire la théorie narrative visant à cerner cette instance problématique, tel que je me suis moimême prêtée à le faire en distinguant inconscience et volonté de la narration pour montrer comment chacune des hypothèses fermées est en mesure de supporter une lecture rentable du Black Note; penser cela donc lire ainsi - nous éloigne d'une expérience autrement logique qui consiste à admettre qu'une narration est un mouvement. Un espace en transformation subissant des variations. Ni constant ni cohérent, un narrateur n'est jamais pleinement fou ni totalement mesquin. Becky Sharp, dans La Foire aux vanités, est peut-être, après tout, aussi manipulée que manipulatrice, prise avec des pulsions contraires. L'image que le lecteur se fait des 
personnages, dans tout roman, est une sorte de tache d'encre tend à s'étendre au fil de la lecture, parfois jusqu'à l'aveugler. Cela est dû au fait que le lecteur détient, au fond, très peu d'informations au sujet des personnages, dévoilés par petites touches et beaucoup de «non-dits ». Qui plus est, l'espace du récit est délimité; il suppose un univers fictionnel sans cloisons, mais paradoxalement, pour ce faire, impose ses propres contours.

Cette complexité de la narration non fiable, vouée au paradoxe et à la contradiction, incite certainement la lecture à se radicaliser. Forcé d'admettre que, dans les aléas du récit non fiable, notamment dans Le Black Note, il est incapable de valider ses hypothèses, le lecteur se sent floué. Sa seule contrepartie, croirait-on, est le caractère décisif (et décidé) de son interprétation. Pourtant, il peut aussi, sans s'avouer vaincu par le texte, chercher à ne pas donner de réponse, mais plutôt à composer avec l'indécidable. Ce serait l'équivalent d'un participant à l'expérience de Luchins qui, torturé par la complexité de sa réponse, choisirait d'annuler « son vote ", de ne pas répondre à la question. Forcément, cette position est inconfortable et ne résout rien (dans $L e$ Black Note, notamment, elle ne résout pas l'affaire criminelle), mais elle a le mérite de souffrir la nuance.

Le même problème se pose à propos du Black Note que dans le Meurtre de Roger Ackroyd; les deux romans n'arrivent pas, faute de narration crédible, à convaincre par la preuve : le narrateur est-il coupable du meurtre ou non coupable? Pour pousser l'expérience de lecture au conflit, il faut accepter que le docteur Sheppard est à la fois coupable du meurtre de Roger Ackroyd et innocent; que le narrateur du Black Note a bel et bien mis le feu à la maison sur l'île et n'a jamais craqué une allumette. En l'absence de réponse aux trous laissés par le texte, le lecteur est invité à rester au carrefour des lectures conflictuelles, là où son regard, plutôt que de se figer, est forcé, malgré l'instabilité que cela implique, d'embrasser l'étendue des possibilités. On voudrait croire, de fait, que la « marque d'une intelligence de premier ordre, c'est la capacité d'avoir deux idées opposées présentes à l'esprit, en même temps, et de ne pas cesser de fonctionner pour autant $\underline{\underline{6}}$ » (Fitzgerald, 1981: 475-476.)

1. Il existe un nombre important de typologies visant à préciser la figure du narrateur ou à faire un pas de côté par rapport à la notion de non-fiabilité narrative. Pensons notamment à la distinction proposée par Ansgar Nünning entre la non-fiabilité relevant de « normes morales » et la non-fiabilité qui ébranle la « véracité factuelle » (2005: 93). Susan S. Lanser fait la différence entre « narrateur non fiable » et «narrateur indigne de confiance » (1981: 171), tandis que Greta Olson préfère opposer « faillible » et « indigne de confiance » (2003: 96). Shlomith Rimmon-Kenan pointe trois cas de narrations non fiables, l'une relevant de “ connaissances lacunaires "; l'autre, d'un « engagement personnel », puis la dernière, d'un "système de valeur problématique » (1983: 100). Chez William Riggan, on retrouve les figures du « narrateur picaresque », de «l'idiot », du « clown » et du « naïf » (1981), qui ont chacun leurs critères de dissimulation. Dorrit Cohn (2000) distingue les narrateurs non fiables des narrateurs discordants. Per Krogh Hansen s'intéresse, quant à lui, aux méthodes de détection de la non-fiabilité, qui sont « intranarratives ", « internarratives », « intertextuelles » ou « extratextuelles » (2007). Ce n'est là qu'un portrait sommaire des catégorisations auxquelles a donné lieu la notion si fuyante de non-fiabilité. [Je traduis.]

2. VIEL, Tanguy. 1998. Le Black Note. Paris : Minuit, p. 23.

3. Le narrateur du Black Note s'adresse-t-il aux allocutaires ou au lecteur, demande Frank Wagner dans « C'est à moi que tu parles? » (2005). S'adresse-t-il seulement à quelqu'un, pourrait-on ajouter. 
4. VIEL, Tanguy. 1998. Le Black Note. Paris : Minuit, p. 39.

5. VIEL, Tanguy. 1998. Le Black Note. Paris : Minuit, p. 39.

6. [Je traduis.]

\section{Bibliographie}

BAYARD, Pierre. 1998. Qui a tué Roger Ackroyd? Paris : Minuit, «Paradoxe », 169 p.

Воотн, Wayne C. 1961. The Rhetoric of Fiction. Chicago : University of Chicago Press, $455 \mathrm{p}$.

Butor, Michel. 1956. L'emploi du temps. Paris : Minuit, 299 p.

CoHn, Dorrit. 2000. «Discordant Narration». Style, vol. 34, no 2, été, p. 307-316.

CHRISTIE, Agatha. 1992 [1926]. Le meurtre de Roger Ackroyd, traduit par Françoise Jamoul. Paris : Éditions du Masque, « Romans d'aventures », 285 p.

Currie, Gregory. 2004. Arts and Minds. Oxford : Clarendon Press, 281 p.

Fitzgerald, F. Scott 1963 [1945]. La Fêlure, traduit de l'anglais par Dominique Aury et Suzanne Mayoux, préface de Roger Grenier. Paris : Gallimard, 364 p.

Fortier, Frances et Andrée Mercier. 2001. «Savoir retenu et savoir manquant. Quelques enjeux de la narration omnisciente dans le récit contemporain », dans Jean-Louis BRAU (dir.), La voix narrative. Nice : Presses universitaires de Nice - Sophia Antipolis, «Cahiers de narratologie », p. 445-460.

HANSEN, Per Krogh. 2007. «Reconsidering the Unreliable Narrator ». Semiotica, no 165, p. 227-246. $<$ https://www.degruyter.com/view/j/semi.2007.2007.issue-165/sem.2007.041/sem.2007.041.xml>.

ISER, Wolfgang. 1978. The Act of Reading. A Theory of Aesthetic Response, traduit par Der Akt des Lesens. Baltimore : John Hopkins University Press, 239 p.

JOUVE, Vincent. 2006. «Les métamorphoses de la lecture narrative». Protée, vol. 34, no 2-3, automne-hiver, p. $153-161$.

LANSER, Susan S. 1981. The Narrative Act. Point of View in Prose Fiction Princeton : Princeton University Press, $308 \mathrm{p}$.

NünNING, Ansgar F. 2005. «Reconceptualizing Unreliable Narration. Synthesizing Cognitive and Rhetorical Approaches », dans James Phelan et Peter J. Rabinowitz (dir.), A Companion to Narrative Theory. Oxford : Malden - Blackwell Publishing, p. 89-107.

Oıson, Greta. 2003. «Reconsidering Unreliability. Fallible and Untrustworthy Narrators». Narrative, vol. 11, no 1, janvier, p. 93-109.

PerRY, Menakhem. 1979. «Literary Dynamics. How the Order of a Text Creates Its Meanings ». Poetics Today, vol. 1, no 1, automne, p. 35-64.

Riggan, William. 1981. Picaros, Madmen, Naïfs, and Clowns. The Unreliable First-Person Narrator 
Norman : University of Oklahoma Press, 206 p.

Rimmon-Kenan, Shlomith. 1983. Narrative Fiction. Contemporary Poetics. Londres; New York : Methuen, $192 \mathrm{p}$.

SAINT-GelaIs, Richard. 1994. Châteaux de pages. La fiction au risque de sa lecture Lasalle (Québec) : Hurtubise HMH, « Brèches », 299 p.

SAINT-GeLAIS, Richard. 1997. «Rudiments de lecture policière ». Revue belge de philologie et d'histoire. Langues et littératures modernes, vol. 75, no 3, p. 789-804.

SternBerg, Meir. 1978. Expositional Modes and Temporal Ordering in Fiction Baltimore : John Hopkins University Press, $338 \mathrm{p}$.

THACKERAY, William. 1994 [1846]. La foire aux vanités, édition annotée et présentée par Sylvère Monod, texte traduit par Georges Guiffrey. Paris : Gallimard, «Folio classique », 1071 p.

VIEL, Tanguy. 1998. Le Black Note. Paris : Minuit, 128 p.

VIEL, Tanguy. 2001. L'Absolue perfection du crime. Paris : Minuit, $176 \mathrm{p}$.

VIEL, Tanguy. 2006. Insoupconnable. Paris : Minuit, 144 p.

WAGNER, Frank. 2005. “C'est à moi que tu parles?" Allocutaires et auditeurs dans Le Black Note de Tanguy Viel », dans Sjef Houppermans, Christine Bosman Delzons et Danièle de RuYTeR-Tognottı (dir.), Territoires et terres d'histoires. Perspectives, horizons, jardins secrets dans la littérature française d'aujourd'hui. Amsterdam; New York : Rodopi, « Faux titre », p. 217-244. 